\title{
Luminosities and mass-loss rates of Local Group AGB stars and Red Supergiants
}

\author{
Martin A. T. Groenewegen ${ }^{* a}$ and Greg C. Sloan ${ }^{b}$ \\ ${ }^{a}$ Koninklijke Sterrenwacht van België Ringlaan 3, B-1180 Brussel, Belgium \\ ${ }^{b}$ Cornell University, Center for Radiophysics \& Space Research Ithaca, NY 14853-6801, USA \\ E-mail: martin.groenewegen@oma.be, sloan@isc.astro.cornell.edu
}

\begin{abstract}
Dusty radiative transfer models are presented for 210 carbon stars and 166 oxygen-rich evolved stars in the Magellanic Clouds and other nearby dwarf galaxies for which Spitzer IRS spectra are available. The spectra are complemented with available optical and infrared photometry to construct spectral energy distributions. A dust radiative transfer code and a minimisation procedure are used to determine the best-fitting luminosity and mass-loss rate. This presentation focuses on the mass-loss rates determined for the carbon stars.

The potentially most interesting result is that a significant number of stars, about $5 \%$ of the sample, has a mass-loss rate that translates to a value of $\beta \equiv\left(\dot{M} v_{\exp }\right) /(L / c)$ (the ratio of the mattermomentum flux to the photon-momentum flux) which is larger than the single-scattering limit and up to a value of $\sim 10$. If confirmed, this result would impact stellar evolution models which sometimes limit the mass-loss rate to $\beta=1$.
\end{abstract}

A full paper will follow with a more complete presentation for the larger sample.

The Life Cycle of Dust in the Universe: Observations, Theory, and Laboratory Experiments - LCDU 2013, 18-22 November 2013

Taipei, Taiwan

*Speaker. 


\section{Introduction}

Almost all stars with initial masses in the range $\sim 0.9-8 \mathrm{M}_{\odot}$ will pass through the asymptotic giant branch (AGB) phase, which is the last stage of active nuclear burning before they become post-AGB stars, planetary nebulae and finally white dwarfs. Slightly more massive stars will pass through the red supergiant (RSG) phase before meeting their likely fates as supernovae. In both cases, mass-loss dominates the final evolutionary stages of the star.

The mass-loss process has been studied in detail in Galactic sources with the advent of the Infrared Astronomical Satellite and the Infrared Space Observatory, but uncertainties in distances lead to uncertainties in luminosities and mass-loss rates (MLRs). Sources at known distances, as in the Large and Small Magellanic Clouds (LMC and SMC), or nearby dwarf spheroidal galaxies (dSphs), reduce this problem, and also allow one to study the effect of metallicity on the MLR.

Groenewegen et al. [1] modelled the spectral energy distributions (SEDs) and spectra from the Infrared Spectrograph (IRS; [2]) onboard the Spitzer Space Telescope [3] for a sample of 60 carbon (C) stars. Groenewegen et al. [4] extended this effort to $101 \mathrm{C}$ stars and 86 oxygen-rich AGB stars and RSGs (hereafter referred to as M stars for simplicity) in the MCs. The cryogenic phase of the Spitzer mission has ended, and all low-resolution IRS spectra are publically available, e.g. through the CASSIS ${ }^{1}$ service [5]. In the present paper we aim to investigate a much larger sample of AGB stars and RSGs in the MCs.

To only consider stars with IRS spectra naturally limits the number of stars for which massloss rates and luminosities may be determined, but aids significantly in the chemical classification of the objects, especially when the sources are obscured and no classification from optical data is available. A thorough analysis of the SEDs and IRS spectra of the nearly 370 AGB stars and RSGs in the MCs is in preparation ( [6], hereafter the "full paper"). Here we focus on a discussion of the mass-loss rates of the $\mathrm{C}$ stars.

\section{The sample and the data}

Several observing programs have obtained Spitzer IRS data of evolved stars in the MCs. The present sample is based on publically available data from the following programmes: 200 (P.I. J. Houck), 3277 (P.I. M. Egan), 3426 (P.I. J. Kastner), 3505 (P.I. P. Wood), 3591 (P.I. F. Kemper), 30788 (P.I. R. Sahai), 40159 (P.I. A. Tielens), 40650 (P.I. L. Looney), 50167 (P.I. G. Clayton), and 50240 (P.I. G. Sloan).

Not all of these programs exclusively observed AGB stars and RSGs. Following [4], targets were selected from these programs by examining the IRS spectra, collecting additional photometry, consulting SIMBAD and the papers describing these programs, and considering the results of the radiative transfer modelling. The present sample consists of $212 \mathrm{C}$ stars and $166 \mathrm{M}$ stars. In addition, a sample of $19 \mathrm{C}$ stars in the Sculptor, Carina and Fornax dSphs were included from program 20357 (P.I. A. Zijlstra; see [7]).

The spectra considered here were obtained with the low-resolution modules of the IRS: ShortLow $(5.1-14.2 \mu \mathrm{m})$, and Long-Low $(14.0-37.0 \mu \mathrm{m})$. Both modules have a resolution $(\lambda / \Delta \lambda)$ of

\footnotetext{
${ }^{1} \mathrm{http}: / /$ cassis.astro.cornell.edu
} 
$\sim 60-100$. For all stars additional broad-band photometry ranging from the optical to the mid-IR was collected from the literature. See the full paper for further details.

\section{The model}

The models are based on the "More of DUSTY" (MoD) code [8], which uses a slightly updated and modified version of the DUSTY dust radiative transfer code [9] as a subroutine within a minimization code. The code determines the best-fitting dust optical depth and luminosity by fitting the photometric data and the spectra.

Those portions of the IRS spectra with poor $\mathrm{S} / \mathrm{N}$ or those affected by background subtraction problems, or that are affected by strong molecular features (which were not included in the simple model atmospheres) are masked and excluded in the minimisation procedure. The photospheric models for $\mathrm{C}$ stars are from [10], while the M stars are modelled by MARCS stellar photospheres [11]. The dust around the $\mathrm{C}$ stars is assumed to be a combination of amorphous carbon (AMC), silicon carbide $(\mathrm{SiC})$, and magnesium sulfide $(\mathrm{MgS})$. The oxygen-rich chemistry is richer and involves more species, as described in more detail in the full paper. A single grain size of $0.2 \mu \mathrm{m}$ has been adopted. The absorption and scattering coefficients are based on a distribution of form factors (see [12], [13]) close to a classical continous distribution of ellipsoids. This distribution gives a reasonably to good fit to the shape of the $30 \mu \mathrm{m}$ feature.

\section{Mass-loss rates}

Figure 1 shows the derived MLRs for the $212 \mathrm{C}$ stars and compares them to synthetic evolutionary tracks based on the models of [14] (hereafter VW93; see [4] for a discussion of these models). Compared to [4] the qualitative description of the comparison has changed. In [4] only three $\mathrm{C}$ stars were slightly above the single-scattering limit, which was considered to be consistent with expectations. [15] studied a set of 900 dynamical model atmospheres for carbon stars with solar metallicities, and they found that the 98th percentile on $\beta \equiv\left(\dot{M} v_{\exp }\right) /(L / c)$ (the ratio of the matter-momentum flux to the photon-momentum flux) is 3.0. From dynamical model atmospheres for subsolar metallicities by [16] one might expect the value for $\beta$ to be a factor 2.6 lower in the LMC, i.e. near unity.

The current picture based on the larger sample is very different. A significant number of $\mathrm{C}$ stars are above the single-scattering limit by up to a factor of 10 . This could be due to uncertainties in the MLR (and consequently $\beta$ ), due to the assumption of a gas-to-dust ratio of 200 and an expansion velocity of $10 \mathrm{~km} \mathrm{~s}^{-1}$, and uncertainties in the dust absorption coefficients. Second, the models of [15] show that $\beta>1$ can be reached in realistic models. If confirmed however, our results show that the artificial cut-off in the models by VW93 at $\beta=1$ is too conservative. A cut-off (if any) at a larger $\beta$ would result in shorter AGB lifetimes!

The right hand panel briefly attempts to address the question of any obvious metallicity dependence of the MLRs. It presents the results in the left-hand panel differently, with the MLRs of the $\mathrm{C}$ stars binned and averaged. In addition to the SMC, LMC and a few dSph stars, we included MLRs derived for Galactic stars (from [17]). At first glance, these samples do not show an obvious dependence of MLR on metallicity, but a more careful analysis will be presented in the full paper. 


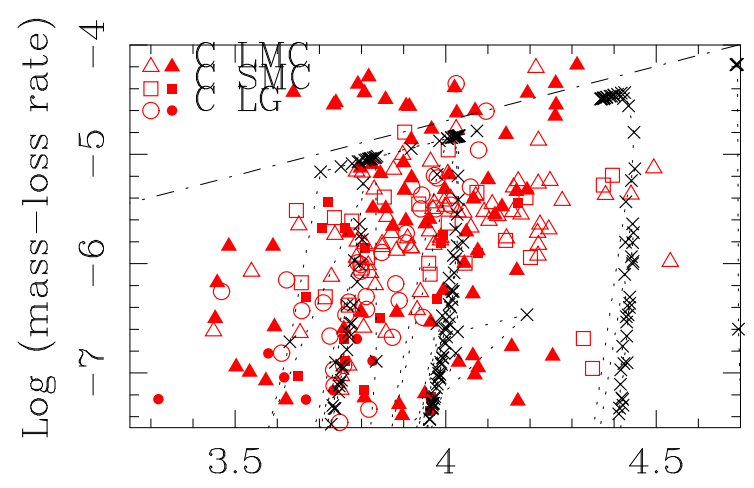

$\log (\mathrm{L})$

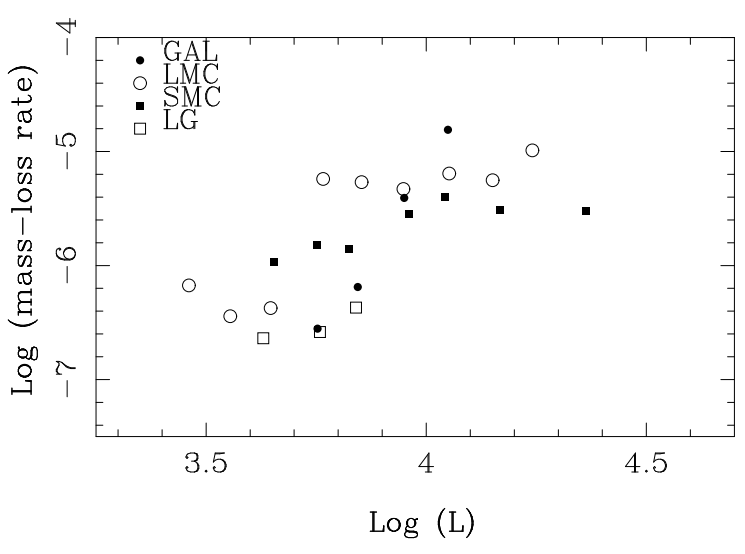

Figure 1: MLR versus luminosity for C stars. Left panel. All stars are plotted, with the symbol as given in the legend. Objects with Mira like pulsation amplitudes are plotted with open symbols, objects with smaller amplitudes or no information on pulsation as filled symbols. The VW93 models are plotted as crosses connected by the dotted line for initial masses of 1.5, 2.5, 5.0 and 7.9 $\mathrm{M}_{\odot}$. Each cross represents a time interval of 5000 years. The dot-dashed line indicates the single scattering limit for a velocity of $10 \mathrm{~km} \mathrm{~s}^{-1}$. Right panel. Binned version of the panel on the left, and including Galactic C stars. There is no obvious dependence of MLR with metallicity.

\section{References}

[1] Groenewegen, M. A. T., Wood, P. R., Sloan, G. C., et al. 2007, MNRAS 367, 313

[2] Houck J. R., Roelling T. L., van Cleve J., et al. 2004, ApJS, 154, 18

[3] Werner M. W., Roellig T. L., Low F. J., et al. 2004, ApJS, 154, 1

[4] Groenewegen M. A. T., Sloan G. C., Soszyński I., \& Petersen E. A. 2009, A\&A, 506, 1277

[5] Lebouteiller, V., Barry, D. J., Spoon, H. W. W., et al. 2011, ApJS, 196, 8

[6] Groenewegen M. A. T., Sloan G. C. 2014, in preparation

[7] Sloan, G. C., Matsuura, M., Lagadec, E., et al. 2012, ApJ, 752, 14

[8] Groenewegen, M. A. T. 2012, A\&A 543, A36

[9] Ivezić, Ž., Nenkova M.,\& Elitzur M. 1999, DUSTY user manual, University of Kentucky report

[10] Aringer, B., Girardi, L., Nowotny, W., Marigo, P., Lederer, M. T. 2009, A\&A, 503, 913

[11] Gustafsson, B., Edvardsson, B., Eriksson, K., et al. 2008, A\&A, 486, 951

[12] Min, M., Hovenier, J.W., Dominik, C., de Koter, A., \& Yurkin, M. A. 2006, JQRST, 97, 161

[13] Mutschke, H., Min, M., \& Tamanai, A. 2009, A\&A, 504, 875

[14] Vassiliadis, E., \& Wood, P. R. 1993, ApJ, 413, 641

[15] Mattsson, L., Wahlin, R., \& Höfner, S. 2010, A\&A, 509, A14

[16] Wachter, A., Winters, J.M., Schröder, K.-P., \& Sedlmayr, E. 2008, A\&A, 486, 497

[17] Groenewegen, M. A. T., Whitelock, P. A., Smith, C. H., \& Kerschbaum F. 1998, MNRAS, 293, 18 